analysis of aneurysms compared to normal arteries is difficult because of risks associated with tissue collection. Endovascular biopsy is a low-risk alternative method for collecting endothelial cells from aneurysms and comparing them to the same patient's non-aneurysmal endothelial cells.

Methods A patient with a large, fusiform, partially thrombosed vertebrobasilar artery aneurysm was recruited for research study after institutional review board approval. We used simple endovascular techniques to collect cells from the patient's vertebrobasilar artery aneurysm and their femoral artery during flow-diverting stent placement. The biopsied cells underwent fluorescence-activated cell sorting (FACS) to isolate viable endotheilal cells. Then, RNA was extracted and cDNA libraries generated using the Smart-seq2 protocol on the Fluidigm C1 platform, followed by sequencing using a Illumina HiSeq2500. Gene expression levels were calculated and filtered as read per kilobase per million mapped reads (RPKM). Principal component analysis, differential gene expression analysis, gene ontology and biological pathway analysis was performed to identify candidate genes and expression pathways related to disease pathogenesis and potential medical therapy.

Results Flow-diverting stents were placed in the ipsilateral vertebral artery, and the contralateral vertebral artery was occluded using coils. The vertebrobasilar aneurysm continued to grow and the patient suffered worsening mass effect on the brainstem before entering hospice care. Gene expression analysis identified significant differences in human leukocyte antigen gene complex series (HLAs) and interferon signaling exonuclease genes, implicating a strong role for the immune system in the progression of this patient's aneurysms. In parallel, the patient underwent occipital artery to anterior inferior cerebellar artery (AICA) bypass to treat a separate AICA aneurysm. Histologic evaluation of a resected occipital artery segment demonstrated necrotizing transmural vasculitis compatible with polyarteritis nodosa.

Conclusion Endovascular biopsy can be performed to obtain aneurysmal tissue with low risk. Analysis of the collected endothelial cells may yield insight into disease pathogenesis and therapeutic alternatives.

Disclosures K. Narsinh: None. K. Narsinh: None. D. McCoy: None. Z. Sun: None. E. Winkler: None. A. Abla: None. D. Cooke: None.

\section{E-013 MIXING ETHANOL AND CONTRAST FOR USE AS A LIQUID EMBOLIC AGENT}

S Rudkin*. Radiology, Allegheny Health Network, Pittsburgh, PA

\subsection{6/neurintsurg-2020-SNIS.49}

Introduction There is currently minimal information in the literature defining the interactions of ethanol with various nonionic contrast agents. There are two main questions to explore: 1) at what concentration ratio will precipitation occur? And 2) does the dilutional effect of contrast reduce the embolic effect of ethanol? The aim of this project is solely to answer the first question.

At our institution, iopamidol (Isovue) and iohexol (Omnipaque) are the main non-ionic contrast agents of choice. We plan to conduct a simple experiment where we will add sequential amount of absolute ethanol to these non-ionic agents until we reach the crystallization threshold concentration. This will provide useful information for the interventionalist such that concern for intracatheter or proximal vessel precipitation can be avoided when using ethanol as a sclerosant for vascular malformations.

Absolute ethanol embolization has been used for the treatment of brain and other AVMs such as those involving the mandible. Ethanol directly damages the endothelial layer and fractures the vascular wall to the level of the internal elastic lamina, which provides a more curative and permanent effect. As a fluid agent, ethanol penetrates to the capillary level. Inflow occlusion with balloon catheters may be required to induce decreased flow and vascular stasis such that full contact of ethanol with endothelium is obtainable. Given its penetrance to the capillary level, all collateral circulation is shut down and necrosis can occur. The amount of ethanol is dependent on the flow and volume characteristics with no set pre-determined amount. The amount of contrast needed to displace blood and not reflux provides a good estimate of the amount of ethanol required for embolization.

Methods We continuously added small amounts of pure ethanol $(98 \%$ ethanol) at a rate of $.15 \mathrm{~mL}$ ethanol $/ \mathrm{min}$ using a syringe pump to a vial containing $3 \mathrm{~mL}$ of Isovue 300 contrast agent. A spinning magnetic stirrer was used in the vial of Isovue to aid in mixing. We monitored for particle precipitation utilizing the Tyndall effect by shining light through the vial.

Results/Discussion There was no evidence for particle precipitation with concentrations reaching as high as $75 \%$ ethanol ( 9 $\mathrm{mL}$ of ethanol/3 mL of Isovue 300). This concentration is even higher than an optimal concentration of 50\% ethanol/ $50 \%$ Isovue, which would provide adequate visualization of the sclerosing agent, while also maintaining its destructive properties.

Conclusion We conclude that adding ethanol to Isovue provides a safe visible liquid embolic agent able to reach the capillary level without causing microcatheter occlusion or proximal artery occlusion from precipitation.

Disclosures S. Rudkin: None.

\section{E-014 ENDOVASCULAR ENDOTHELIAL BIOPSY OF INTRACRANIAL ANEURYSMS FOR PERSONALIZED RISK STRATIFICATION USING SINGLE CELL RNASEQ}

${ }^{1} \mathrm{C}$ Young ${ }^{*},{ }^{1,2} \mathrm{~S}$ Levy, ${ }^{1} \mathrm{~A}$ Patel, ${ }^{3} \mathrm{D}$ Cooke, ${ }^{4} Y$ Zheng, ${ }^{4} \mathrm{C}$ Mandrycky, ${ }^{1,2} \mathrm{C}$ Kelly, ${ }^{1,2} \mathrm{~L}$ Kim, ${ }^{1,2} \mathrm{M}$ Levitt. ${ }^{1}$ Neurological Surgery, University of Washington, Seattle, WA; ${ }^{2}$ Stroke and Applied Neurosciences Center, University of Washington, Seattle, WA; ${ }^{3}$ Radiology and Biomedical Imaging, University of California, San Francisco, San Francisco, $C A_{\text {; }}$ ${ }^{4}$ Bioengineering, University of Washington, Seattle, WA

\subsection{6/neurintsurg-2020-SNIS.50}

Introduction Early detection and treatment of intracranial aneurysms can prevent hemorrhagic stroke. However, treatment requires an invasive surgical procedure, which is potentially risky and costly. Risk quantification systems based on aneurysm size are imperfect since the majority of ruptured aneurysms are small, and no robust method exists for predicting an individual aneurysm's likelihood of rupture. Endothelial cell (EC) genetics have been proposed to play a critical role in aneurysm risk. However, with the advent of endovascular treatment of aneurysms, there is a decline in the availability of biological tissue for research. 